\title{
Large-Scale Simulation and Visualization in Medicine: Applications to Cardiology, Neuroscience, and Medical Imaging
}

\author{
Chris Johnson \\ Director, Scientific Computing and Imaging Institute \\ School of Computing \\ Merrill Engineering Building \\ 50 South Campus Central Dr., Room 3490 \\ University of Utah \\ Salt Lake City, Utah 84112-9205 \\ Phone: +1-(801)-585-1867 \\ FAX: +1-(801)-585-6513 \\ crj@cs.utah.edu
}

\begin{abstract}
Computational problems in medicine often require a researcher to apply diverse skills in confronting problems involving very large data sets, three-dimensional complex geometries which must be modeled and visualized, large scale computing, and hefty amounts of numerical analysis. In this talk I will present recent research results in computational neuroscience, imaging, and cardiology. I will provide examples of several driving applications of steering and interactive visualization in cardiology (defibrillation simulation and device design), neuroscience (new inverse source localization techniques), and imaging (new methods for interactive visualization of large-scale 3D MRI and CT volumes, and introduce new methods for diffusion tensor imaging).
\end{abstract}

